\title{
Erratum to: Different expression profiles of jasmonic acid and salicylic acid inducible genes in the tomato plant against herbivores with various feeding modes
}

\author{
Kei Kawazu ${ }^{1} \cdot$ Atsushi Mochizuki $^{1} \cdot$ Yukie Sato $^{1} \cdot$ \\ Wataru Sugeno $^{1} \cdot$ Mika Murata $^{1} \cdot$ Shigemi Seo $^{2}$. \\ Ichiro Mitsuhara ${ }^{2}$
}

Published online: 6 January 2016

(C) Springer Science+Business Media Dordrecht 2016

Erratum to: Arthropod-Plant Interactions (2012)

6:221-230

DOI 10.1007/s11829-011-9174-z
The authors would like to apologize for this error.

The correct version of Table 1 is given below.

Unfortunately, there is an error in the Table 1 in the original publication of the article.

Table 1 Genes and primers used for real-time PCR amplification

\begin{tabular}{lll}
\hline Gene & Forward primer: sequence $\left(5^{\prime}-3^{\prime}\right)$ & Reverse primer: sequence $\left(5^{\prime}-3^{\prime}\right)$ \\
\hline Pin2 & CATCTTCTGGATTGCCCA & ACACACAACTTGATGCCCAC \\
LapA1 & GGGACTAATGATGTTTGGAA & GTGGCAATTTTATTTAGGCA \\
$P R b-1 b$ & TTTCCCTTTTGATGTTGCT & TGGAAACAAGAAGATGCAGT \\
GluB & CCTTTTGTTTGTCATTCTGG & GCAGTTACCAGATCAGAGTGT \\
Actin & CCAGGTATTGCTGATAGAATGAG & GAGCCTCCAATCCAGACAC \\
\hline
\end{tabular}

The online version of the original article can be found under doi:10.1007/s11829-011-9174-z.

\section{Atsushi Mochizuki}

lepi@affrc.go.jp

1 National Institute for Agro-Environmental Sciences,

3-1-3 Kannondai, Tsukuba, Ibaraki 305-8604, Japan

2 National Institute of Agrobiological Sciences,

2-1-2 Kannondai, Tsukuba, Ibaraki 305-8602, Japan 